\title{
Exploring use of incorrect terminology used in medical sciences: quest for scientific and academic verity
}

\author{
Nasir Abdul Latif Sarwani ${ }^{1}$, Aysha Fatima ${ }^{1}$, Henry James ${ }^{1}$, Hala Ebrahim A.Aziz AlMehzaa ${ }^{2}$, \\ *Manoj Chakravarty ${ }^{1}$ \\ 1. College of Medicine \& Medical Sciences, Arabian Gulf University, Bahrain \\ 2. Ministry of Health, Master Health Policy \& Population Studies, Bahrain \\ * E-mail of the corresponding author: manojc@agu.edu.bh
}

\begin{abstract}
Scientific terminology is used in the context of academic and clinical settings and scientists create novel terms to name them. Many of such terms used in basic and clinical sciences nevertheless appear flawed, and have remained unexamined for a long period of time. Due to common usage, such inappropriate terms have gradually become part of the common language of medical science and continue to be in use. Terms should reflect scientific brevity, be self-explanatory, overcome ambiguity, provide for universal usage, and help basic science to integrate better with the clinical domain logically, correctly and practically. Despite existing efforts in standardization, a large number of non-conforming terms appear to remain in medical use. Some of these are carried on from older terminology, and others are simply the result of convenient habits and usage albeit their logical inconsistency. Words with well-known meaning in common language often pose an academic challenge due to inconsistencies in nomenclature. This study which is the first of its kind, aims at questioning a plethora of terms currently being used in the disciplines of Anatomy, Radiology, Medicine and Surgery that are inappropriate, both logically as well as scientifically, and recommends that these be dropped from the inventory of terms used in medical sciences, and replaced with terminology presented in this article that are semantically logical, scientifically valid, as well as practical.
\end{abstract}

Keywords: Medical terminology, Anatomical terminology

DOI: $10.7176 / J E P / 10-6-19$

"There is little doubt that many concepts which are at present accepted and in general use will in the future have to be considerably modified or even abandoned". - A. Brodal, Oslo, 1968.

\section{Introduction}

Historically, the ancient Greeks were the first to delve into the field of medicine including anatomy, and develop a suitable vocabulary. With the Romans establishing supremacy thereafter, Latin continued to prevail as the language of science throughout Western Europe until the eighteenth century. As a result, a significant number of scientific terms in medical literature derived directly from their Latin roots, and their subsequent transliteration into English have not been scientifically accurate. Sound application of terminology reduces ambiguity and increases clarity, and terminology can thus be a safety factor, a quality factor and a productivity factor in its own right. Terminology is concerned with the correct relationship between concepts, and between them and what they represent and there is no universal agreement as to the meaning of the terms among scientists and between texts. Hence, if standard interpretations of scientific knowledge to be taught do not actually exist, then how can the teaching of standard interpretations of scientific knowledge be established as a goal to be accomplished? (Slisko, 1997).

Terminology serves as a basic communication tool in all areas of the medical field and it is notable to state in context that there are terms devoid of scientific fidelity which continue to be used in medical practice including textbooks. A list of such terms is being presented in this article with scientific and logical arguments to refute them, and recommendations for new and appropriate terminology are being offered simultaneously to replace these terms with modified ones based on scientific analogy, logical argument and evidential support. The list is by no means complete, and more terms need to be identified, rectified or replaced in future. Terms that are identified as questionable and flawed are listed in this article in alphabetical sequence for the purpose of ease of literary flow, and recommended amendments using alternative terminology to replace such terms using scientific brevity are hereby proposed. 


\section{Result}

2.1 Abdominal visceral ligaments: These are relatively thin sheets of peritoneum that connect visceral organs to the abdominal walls and to one another and facilitate the transmission of blood vessels, nerves and lymphatics between them and the abdominal walls. These peritoneal extensions do not possess the structural and histological characteristics of musculo-skeletal ligaments and thus it would be prudent to seek an alternative and scientific terminology that is based on embryological and structural foundations. These sheets are in reality remnants of the embryonic dorsal and ventral mesogastria and persist as mesenteries. In light of these observations, the term gastro-hepatic ligament would be appropriately replaced with the term 'gastro-hepatic mesentery', the gastrosplenic ligament with the term 'gastro-splenic mesentery', and the falciform ligament with the term 'hepatoabdominal mesentery' or 'hepato-umbilical mesentery'.

2.2 Acquired immune deficiency syndrome (AIDS): It is seemingly apparent that AIDS is most certainly not a clear-cut description of any definite medical syndrome, and there does not exist even a single scientific definition of "AIDS" on which discourse can be reliably based. It is clear that the syndrome described as AIDS is vague and its use in medical practice is inappropriate, confusing and unscientific. The term Acquired immune deficiency syndrome or AIDS used worldwide, is not a specific syndrome but is an amalgamation of over twenty diverse and mutually unrelated medical conditions referred to as 'AIDS defining illnesses' that are known to exist independently without any relation to HIV infection. It is the presence and correlation with HIV that is the key factor in this context, and use of the term Acquired immune deficiency syndrome or the acronym AIDS as an established and well-defined syndrome is incorrect. It has been proposed that the term AIDS be abandoned and replaced by the terms "HIV related" or "HIV associated" illnesses (Chakravarty, 2018).

2.3 Angio-: The prefix angio- as used in terms such as angiography and angioplasty is generic and includes the arterial as well as the venous components of the vascular system without differentiating between them. When referring to the arterial domain, the prefix 'arterio' needs to be used, and when referring to the venous domain the prefix 'veno' needs to be used. Thus, the terms 'arteriography', 'venography', 'arterioplasty' and 'venoplasty' are more exacting and scientific than use of the generalized prefix 'angio' in context.

2.4 Autonomic nervous system: Use of the term 'nervous system' in this context presumes that the system is made up of efferent as well as afferent components when in reality, the autonomic subdivision of the nervous system is a purely motor system that supplies smooth muscles and glands which apart from the sweat glands, are essentially visceral in extent and function. It is more appropriate to designate the autonomic nervous system as the 'viscero-motor nervous system' or the 'autonomic motor system'.

Basal ganglia: Ganglia by definition, are clusters of neuron cell bodies located in the Peripheral nervous system whereas nuclei are clusters of neurons located within the Central nervous system (CNS). Use of the term 'ganglia' for collection of neurons within the CNS is incorrect and must be replaced with the terms Basal / Intracerebral / Intracerebellar or Subcortical 'nuclei'.

2.5 Blood supply: The term 'supply' denotes an active anterograde transport of blood which the arterial system provides. However, the concept of 'supply' is not applicable in the case of veins since the venous system facilitates a retrograde transport that is comparatively passive and thus, fails to support the concept of 'supply' in relation to its function. The widely used term of 'blood supply' to denote both arterial as well as venous distributions of the anterograde and retrograde vascular transport respectively is incorrect, and needs to be referred to as 'vascularity' only. The anterograde flow of blood from the heart to the periphery needs to be designated as 'arterial supply', and the retrograde flow of blood from the periphery to the heart must be termed as 'venous return' or 'venous drainage'.

2.6 Cancer: The ancients used the word 'cancer' (crab in Latin) to mean a malignancy, because of the crab-like invasive phenomenon a malignant tumour appeared to exhibit by systematically destroying the tissues it invaded. Malignancy (from Latin 'male', meaning 'badly', and 'gnus', meaning 'born') is the tendency of a medical condition to become progressively worse, and is a term for diseases in which abnormal cells divide without control and invade nearby tissues. Malignant cells can also spread to other parts of the body through the blood and lymph systems (NCI Dictionary of Cancer Terms). Cancer is a colloquial term whereas malignant tumours depart from the parent tissues morphologically and functionally to include the features of anaplasia, mitotic activity, growth pattern, invasion and metastasis as its integral features (Rubin's Pathology, 2012). Malignancy is the character and tendency of a medical condition, especially tumours, to become progressively worse and to potentially result in death. It is thus practical as well as logical and scientific to replace the term 'cancer' with the term 'malignant neoplasm' or 'malignancy' wherever applicable.

2.7 Central 'tendon' of the diaphragm: This structure is anatomically, a true aponeurosis that is defined as - "a 
broad flat sheet of dense fibrous collagenous connective tissue that covers, invests, and forms the terminations and attachments of various muscles" (Merriam Webster dictionary). It is also defined as - "a sheet-like tendinous expansion, mainly serving to connect a muscle with the parts it moves" (The Free Dictionary). Its description and identification as a tendon is incorrect. The correct term to be used is the "central aponeurosis" of the diaphragm.

2.8 Constrictions of the oesophagus: The term 'constrictions' with reference to the oesophagus is used universally in textbooks of basic as well as clinical sciences which state that there are four sites or zones of constrictions. These so-called constrictions have no structural modifications or adaptations that are represented by zones of muscular constriction or bands under normal conditions. These are principally zones of compression caused by external factors that described in order are the cricopharyngeus muscle (sphincter), the aortic arch, the left principal bronchus and the site where it passes through the diaphragm, and these zones cannot be referred to as sites or zones of 'constriction'. These sites are therefore better described as 'zones of compression' and not constrictions. Structural changes that might be considered as sites of constriction are found in pathological conditions referred to as 'oesophageal rings' of which three have been described in literature. Type A is a muscular ring located roughly $2 \mathrm{~cm}$ proximal to the squamo-columnar junction and it represents a proliferation of the proximal border of the lower oesophageal sphincter (Goyal, et al, 1971). Type B (Schatzki) is a mucosal ring located at the squamo-columnar junction and is typically associated with the proximal margin of a hiatal hernia (Varadarajulu, 2003). Type $\mathrm{C}$ is an indentation caused by the diaphragmatic crura, sometimes seen on radiographic studies (Tobin, 1998).

2.9 Constrictions of the ureters: Like in the case of the oesophagus, the so-called constrictions of the ureters are compression-zones created by the pressure of surrounding structures at fixed reference sites and are best referred to as 'Normal sites of compression'.

2.10 Death: Terminology used in certifying death - There is confusion regarding use of terms in relation to the certification of the cause of death in medical practice. Terms used in context are, 'cause of death', 'immediate cause of death' and 'underlying cause of death' (Brooks, 2015). The cause-of-death section as per CDC guidelines consists of two parts. Part I is for reporting a chain of events leading directly to death, with the immediate cause of death (the final disease, injury, or complication directly causing death), and the underlying cause of death (the disease or injury that initiated the chain of events that led directly and inevitably to death). Part II is for reporting all other significant diseases, conditions, or injuries that contributed to death but which did not result in the underlying cause of death given in Part I (CDC, 2003). There is also a grey zone that does not effectively address the issue of death due to 'natural' versus death due to 'unnatural' causes. What the recommended guidelines have done is to have replaced the literal or true cause of death with a summary of events leading to death listed under several categories. In the present light, if these entries must be represented under a composite and meaningful title, then the Death Certificate should be re-designated as "Certification of Summary of Events leading to Death" since the medical cause of 'immediate' death in a hospital setting is almost always due to 'cardio-respiratory failure'.

2.11 Dye or Contrast medium: The term 'dye' in relation to imaging is incorrect since the substance used is monochrome (colourless) and does not offer colour as a mode of contrast. The substance used is soluble and transparent but has the property to block x-rays used in Radiography and CT, and MR signal in MR imaging. The substance thus acts as a medium that enhances contrast and is best described as a 'Contrast medium' rather than a dye.

2.12 External sphincters of the urethra: Two urethral sphincters, one internal and the other external have been described and appear in textbooks including the authoritative Gray's Anatomy (1995) which states, "of the two urethral sphincters, the internal sphincter vescicae controls the vescicle neck... and composed of non-striated muscle....and the external sphincter urethrae surrounds the membranous urethra (Gray's Anatomy, 1995). The fact is that the sphincter vescicae is the sphincter of the bladder and is formed by a specialized arrangement of the longitudinal muscles of the bladder wall provides substance to the fact that this is not a sphincter of the urethra. Conversely, what is generally referred to as the external sphincter of the urethra is in reality, the 'sphincter urethrae'. This muscle is considered to be part of the deep transverse perinei muscle of the urogenital diaphragm and is sometimes considered as a separate muscular entity and is the true sphincter of the urethra, both structurally and functionally. This sphincter surrounds the membranous urethra, is skeletal in type and voluntary in function as opposed to the sphincter of the urinary bladder which is formed by smooth muscles of bladder origin, and is involuntary in function. The bladder sphincter located at the vescico-urethral junction is the 'sphincter vescicae' formed by the muscle of the bladder, and referring to the sphincter vescicae as the internal urethral sphincter and the sphincter urethrae as the external sphincter of the urethra is incorrect. There is 
only one urethral sphincter and that is the sphincter urethrae and the use of the term internal and external sphincter of the urethra in context is incorrect, unsubstantiated and requires replacement.

2.13 Hormone replacement therapy (HRT): Symptoms associated with menopause include hot flashes, vaginal atrophy and dryness, and bone loss among others, and are caused by diminished levels of sex hormones in the menopausal period. To provide hormonal support for this condition, hormonal preparations are administered to bring the hormonal status comparable to pre-menopausal levels. This treatment is accurately described as 'menopausal hormone therapy' (MHT) or 'post-menopausal hormone therapy' (PMHT) and the term MHT has been in medical use since the 1990s (Stuenkel et al, 2015), (Santen et al, 2010). It is incorrect to use the term 'hormone replacement therapy' since this regimen does not replace hormones in the body in any way, but supplements, replenishes or augments the dwindling levels of sex hormones following menopause.

2.14 Imaging: X-rays: These are electromagnetic waves and are invisible. To refer to X-ray films or plates as ' $\mathrm{X}$ rays' is scientifically unacceptable. The correct terms to be used are Radiographs, Skiagrams, Roentgenograms or X-ray films, plates and images in the digital format.

2.15 Intervertebral disk abnormalities: 'Hernia' and 'prolapse' are terms that are commonly used to describe a protruded or bulging intervertebral disc and are used universally and interchangeably to describe the posterior displacement of disk elements into the vertebral canal. The term hernia in context is incorrect as the definitions of a hernia do not apply fit to this condition. Definition 1 - A hernia is an outpouching of the parietal peritoneum through a preformed or secondarily established hiatus (Conze, et al, 2001). Definition 2 - A hernia is the abnormal exit of tissue or an organ, such as the bowel, through the wall of the cavity in which it normally resides. (Fitzgibbons RJ, 2015). Definition 3 - A hernia is defined as a protrusion of a viscus through an opening in the wall of the cavity in which it is contained (Shwartz, 1999). The term 'prolapse' is defined as "a condition in which an organ of the body has slipped forward or down so as to out of place" (Oxford Advanced Learner's Dictionary, 1995) and is incorrectly applied to a bulging IV disc. 'Slipped' disc is another common term that's also used interchangeably with the terms herniated and prolapsed disc and is inappropriate as well. With a bulging disc, that is technically characterized as 'disc protrusion', the spinal disc begins to bulge or protrude outward due to excessive and progressive compressive forces from adjacent vertebrae, but it has not yet torn open. Protrusions are defined as the extension of the disc material up to $25 \%$ of the disc beyond its normal boundaries, and extrusions are defined as extension of the disc material up to $25 \%$ of the normal disc boundaries, attached to the central zone by a stalk. Extension of disc material of greater than $25 \%$ of the disc circumference is called disc 'bulge'. Protrusions can also be multifocal (Fardon, 2014). The terms herniation and prolapse are inappropriate. It is therefore technically correct to use the terms 'protrusion' and 'bulging' instead of hernia or prolapse.

2.16 Intra-peritoneal \& retro-peritoneal: These two terms are universally used to describe the peritoneal relationships of abdominal organs. When organs are completely covered by peritoneum and are connected to the abdominal wall by a mesentery, they are termed as intraperitoneal, whereas when organs have peritoneum covering their anterior surfaces only and lack a mesentery, they are termed as retroperitoneal. The reality is that all abdominal organs have an intimate relationship with the peritoneum and all of them are covered by it (whole or in part) and are thus behind (retroperitoneal) it in the logical sense of the term. The basic point of structural difference that is notable is that the intraperitoneal organs have mesenteries and are thus mobile, while the retroperitoneal ones which are actually "extraperitoneal" in location, lack a mesentery and are immobile. Both structural as well as mobility factors are dependent on the mesenteric status of these organs, and thus, use of the terms 'mesenteric' in place of 'intraperitoneal' and 'non-mesenteric' in place of 'retroperitoneal' in relation to abdomino-pelvic organs is more appropriate. On a similar note, the area that is surgically denoted as the retroperitoneum could be more aptly described as the "extraperitoneum".

2.17 Joints: Knee joint - most sources classify the tibio-femoral articulation as a 'hinge' or 'modified hinge' type of joint. In reality, it neither has the morphology, axial nor kinematic characteristics of a hinge joint. It is technically a 'bi-condylar' joint. The bi-condylar tibio-femoral articulation is functionally described as complex or compound. Movements include flexion, extension, rotation and translation in relation to the joint. The joint is classically described as two separate condylar joints that are parallel between the femur and the tibia. (Gray's Anatomy, 1995). There is a higher degree of incongruity at the lateral tibio-femoral articulation as compared to the medial tibio-femoral articulation (Hohe, et al, 2002). The tibio-femoral articular surfaces are incongruent and further detracts from the fact that the joint could be hinge in type as hinge joints are classically congruent in nature, an example being the Humero-ulnar joint. To describe the knee joint as a 'hinge' or even 'modified hinge' is inaccurate. The term 'bicondylar' from the perspective of articular morphology and arthrokinematics, is the accurate term that needs to be used universally and reference to the joint as a hinge or modified hinge must be 
abandoned.

Wrist (radiocarpal) joint - Joints are named according to the morphology of articulating bony surfaces as well as on the basis of functionality. Classification of synovial joints is primarily based on the kinematics of their articular surfaces. The radiocarpal joint is often described as a condyloid joint when in reality, the surface of the distal radio-ulnar articulation is 'ellipsoid', and the reciprocal articular surface of the scaphoid-lunate unit acts as a congruent elliptical convex surface that reciprocates the elliptical surface of the radius.

Subtalar joint - Subtalar joint movements and their axes of rotation are complex. Most textbooks describe the 'subtalar' joint as the joint between the talus and the calcaneus when in actual fact, inversion-eversion arthrokinematics involve additional joints. These are the talo-calcaneal, talo-navicular, calcaneo-cuboid and the functional transverse tarsal (midtarsal) joints that act as a unit, and together, they represent the 'subtalar joint complex'. There are three facets on the calcaneus that articulate with the talus where the anterior and middle facets are concave, and the posterior facet is convex. Due to the convex posterior facet of the calcaneus and corresponding concave facet of the talus, subtalar joint movement can be described as rotation, translation or a combination of both (Sarrafian, 1993). The talo-navicular joint and the three subtalar joints are linked anatomically and functionally (Hicks, 1953) (Seringe, 2013). It is thus more scientifically appropriate to refer to the movements of inversion and eversion of the foot as occurring at the 'subtalar joint complex' rather than at the subtalar joint.

2.18 Joint dislocation: When the participating articular components of a joint lose contact with each other, then the joint they represent is generally referred to as being 'dislocated'. In reality, the articulating bony surfaces in the joint lose their articular congruence, but the joint remains where it is. Thus, the joint itself is not dislocated from its own position in time and space, but internally, the articular surfaces have become displaced with respect to each other. It is the bone that slips out of articular contact that is dislocated, and not the joint. The phenomenon of displacement of an articular surface from its kinematic geometry is best classified as a 'disarticulation' wherein, the disarticulated bony end has 'dislocated' but not the joint which remains where it is. If the term dislocation needs to be used, it must refer to the bone that has dislocated. Thus, the commonly used term 'shoulder dislocation' needs to be replaced by the term 'humeral dislocation at the shoulder'.

2.19 Lobes of the liver: The segmental anatomy of the liver is based upon ramifications of the portal triad structures. On this basis, the liver can be structurally divided into a right and a left lobe of equal size. The plane of division called the Cantlie's line, runs from the inferior vena cava to the middle of the gall bladder fossa (Scott-Conner, 1993) according to the branching pattern of the right and left hepatic arteries based on the studies by Couinaud in 1953, and Hjortsjo in 1956 (Gray's Anatomy, 1995) and as a result, the caudate as well as the quadrate lobes are designated as part of the left lobe. To describe lobation of the liver as anatomical (structural) and physiological (functional) where each one is different from the other is impractical and devoid of logic. There is only one true gross lobation, and this is represented by the Cantlie's line. The only unit in the liver that can be described as a true functional unit however, is histological and is named the "hepatic acinus" which is defined as "the functional unit of the liver that consists a mass of liver tissue cells (hepatocytes) aligned around the hepatic arterioles and portal venules (Schwab, 2011).

2.20 Lumbar puncture: This ill-defined term does not distinguish between a subarachnoid tap, a subarachnoid therapeutic infiltration (anaesthetic or antimicrobial) or an epidural infiltration for anaesthesia. The term lumbar puncture literally means to puncture the lumbar spinal column. The terms 'Lumbar cisternal' or 'sub-arachnoid' tap or injection or block, and 'Lumbar epidural' block are more appropriate terms that need to be used based on which procedure is being done at a given time.

2.21 Mole and molar quantities: The mole is the quantity of particles of any substance that is equal to the Avogadro's number or constant ie. $6.0221 \times 10^{23}$ particles. A mole of a substance contains the same number of elementary particles as there are atoms in exactly 12.000 grams of ${ }^{12} \mathrm{C}$ isotope of Carbon. Each time we use molar quantities, we are expected to possess an insight into their relationships to the number of particles in exactly 12 grams of ${ }^{12} \mathrm{C}$ isotope of carbon. Molar quantities are used to express concentrations of substances in solution in relation to the Avogadro's number. Molarity is a molar quantity of the concentration of the number of moles of a substance that are dissolved in 1 litre of water. Does use of these terms appear to help provide a tangible measure of substances in relation to a given clinical scenario for the student, physician and the patient? The number with a value of $6.0221 \times 10^{23}$, lies in a range which is far beyond all human experience (Logan, 1998). Adding the complication of operating on and interrelating three levels of thought: the macro and tangible, the submicro-atomic \& molecular, and representational use of symbols and mathematics is impractical. It is a psychological folly to introduce learners at all three levels simultaneously. The trained chemist can keep these 
three things in balance, but not the learner. Kept as an extensive property of matter rather than an intensive property of solution, the mole is not a formidable idea (Johnstone, 2000). Stoichiometric relations produce conflicting situations for students, leading to conceptual misunderstanding of concepts, such as mass, atoms and moles (de Astudillo, 1996). Does it provide any tangible value of anything other than comparing number of molecules of a given substance in solution to the number of molecules of ${ }^{12} \mathrm{C}$ isotope of carbon? Use of molar quantities are suitable for chemists \& physicists (and to anyone else who might be interested in stoichiometric analogies), and using this unit in terminology in a hospital and medical school setting is impractical and gives absolutely no idea regarding any finite or tangible appreciation of the quantity of any substance but only represents a numerical value. Use of conventional units such as gram/milligram $/ \mathrm{microgram} /$ picogram/femtogram per deciliter $(\mathrm{gm} / \mathrm{dL}, \mathrm{mg} / \mathrm{dL}, \mu \mathrm{gm} / \mathrm{dL}, \mathrm{pgm} / \mathrm{dL}, \mathrm{fgm} / \mathrm{dL})$ is far more practical in terms of finite and appreciable concentration of any substance directly, and is meaningful, tangible and logical, and needs to be restored as the unit of reference for physicians and students in general and and patients in particular rather than the intangible and impractical academic units of $\mathrm{mmol} / \mathrm{L}$ or $\mathrm{mEq} / \mathrm{L}$ which must be retained only for academic discussion among experts.

2.22 Movements related to joints ('of' or 'at'): Movements 'at' a joint versus movement 'of' a joint - All movements that occur in relation to a joint are with reference to the part of the body which is in motion. Thus, a part of the body moves in relation to a joint which remains fixed in its relative position. So movement is essentially of a part of the body that occurs 'at' the joint. Movement 'of' a joint is the movement of the part of the body where the joint is located. In relation to the shoulder, movements 'at' the joint are the movements of the upper limb or the arm and includes the movements of flexion, extension, adduction, abduction, rotation and circumduction. Movements 'of' the joint denote movements of the shoulder as a unit and are displayed by the movements of protraction, retraction, elevation and depression where the entire joint moves in relation to the body.

2.23 Nerve compression or stretching as the causative factor in intervertebral disc disease: The pathophysiological mechanism for the cause of pain and related symptoms caused by a protruded intervertebral disc on nerve roots is widely described to be compressive and inflammatory in nature. Compression is certainly a factor when it comes to disc protrusion above the level of the L1 vertebra where the spinal cord almost entirely occupies the vertebral canal. Below this level, the vertebral canal is occupied by the nerve roots forming the cauda equina. At this level and below and without the limitational effect of spinal stenosis, central and posterolateral disc protrusions essentially create a bow-stringing (stretching) effect on the nerve roots since more space is available within the canal where the nerve roots have a degree of mobility that is almost absent at vertebral levels above L1 vertebra. There is no clear relationship between the extent of disk protrusion and the degree of clinical symptoms (Haldeman, 1990). A strictly mechanical or patho-anatomical explanation for low back pain and sciatica has proved inadequate. Although the predominance of the existing spine literature has addressed nerve root compression as the principal cause of pain, it is equally likely that a stretch mechanism may be responsible for all or part of the pathology (Kitab, 2009). Mechanical stretching is known to cause morphologic and functional changes in peripheral nerve. Studies have shown that peripheral nerves exhibit nonlinear stress-strain characteristics when placed under tension. Initially, under tension, the nerve has a low modulus that increases gradually with increasing strain until reaching a maximal value. When the nerve fails under tension, the perineurium inside the nerve ruptures, but the exterior of the nerve remains intact. The maintenance of small in-situ stresses suggests that sustained increases in tension could be adversely affecting the electrophysiologic properties of the nerve. Indeed, marked alteration of conduction properties result from even a small stretch of 6 percent beyond the in-situ length of the nerve, or stress less than 10 percent of the ultimate strength of the nerve (Kwan et al, 2009).

2.24 Nerve supply and Innervation: Both terms essentially refer to an anterograde form of signal transport that denotes a 'motor' or effector function. The term innervation a transitive verb and is defined as the process of supplying nerves to an organ or part of the body (Cambridge English Dictionary). Thus, the terms supply and innervation are synonymous. The term 'nerve supply' is incorrect since supply (motor) is an anterograde phenomenon while the functions of sensation or sensibility designate a retrograde phenomenon that cannot be justified by using the term 'supply'. Thus, motor transport of impulses needs to retain the term 'supply' whereas sensory transport of signals that is retrograde, requires re-naming. In this respect, the use of the terms 'efferent' and 'afferent' with respect to motor and sensory functions respectively is valid and needs to be used as such. It is recommended that the terms 'afferent function' and 'efferent function' replace the terms 'nerve supply' and 'innervation' to denote both motor and sensory functions.

2.25 Organ-dynamics in Embryology: These are all relative outcomes influenced by differential growth patterns 
of various organs/structures and the body wall. 'Cranio-caudal migration' and 'Relocation' are more exacting and scientific terms in context.

Descent of the diaphragm: The migration of muscle and projection of nerve to the pleuro-peritoneal folds (PPFs) are key developmental events (Allan, 1997), (Babiuk et al, 2003). The PPFs expand dorsally and ventrally across the septum transversum, a thin membrane on the cranial surface of the liver, and give rise to the diaphragm's muscle connective tissue and central tendon (Merrell et al., 2015). There is some evidence that the PPFs originate in the cervical region (Hirasawa et al., 2016), but nascent PPFs have never been explicitly labelled and their early development tracked. The somites are the source of the diaphragm's muscle, and muscle progenitors emigrate from the somites into the PPFs (Merrell et al., 2015), (Sefton, 2018). The diaphragm thus is better described as having migrated rather than having descended.

Descent of the testes: During the human fetal period, the testes migrate from the lower posterior abdominal wall to the scrotum, traversing the abdominal wall and the inguinal canal between the 15 th and the 28th week post - conception, and several theories have been developed to explain testicular migration (Sampaio, 1998). Testicular migration happens in two distinct phases: the first phase involves testicular migration from the abdomen to the internal inguinal ring, and the second phase involves the transition of the testes through the inguinal canal until their definitive arrival at the scrotum (Hughes, 2008). The term migration is more exacting and suitable for use in context of movements described in relation to the testes.

Pituitary gland: The same logical argument holds ground in respect of the development of the pituitary gland where relative growth patterns bring about the final relationship in structure via intricate steps in migration that are dependent on the relative growth patterns of the head, neck, neural tube and the pharynx. Thus, in relation to the pituitary, there is no real descent of the hypothalamic component and no ascent of the pharyngeal (Rathke's pouch) component. Everything is about relative growth, and the concept of migration is the most appropriate feature that helps to explain the change between origin of growth and its final position in the body.

2.26 Origin and Insertion of muscles: The terms 'origin' and 'insertion' represent a functional description of a muscle's attachments because it names an attachment on the basis of whether or not it moves (Muscolino, 2010). The terms are not recommended by the "Terminologia Anatomica" since 1998. Their recommendation clearly states that the terms origin and insertion are not to be used since they change with function (Federative International Program on Anatomical Terminologies, 2013). The confusion is with respect to which site of attachment is fixed and which site moves during movement of a part of the body, and based on the old concept that the site of the muscle 'origin' is fixed and the site of the 'insertion' moves is not consistent. Thus, the structural relationship with the site of attachment of muscles should be termed as 'attachments' rather than using the terms origin and insertion that are unsubstantiated, dated and incorrect. Sites of attachment are to be named on the basis of the bony part where the muscle is attached eg. scapular, humeral, pelvic, costal etc. or based on the relationship with the points of reference of the body eg. proximal, distal, cranial, caudal etc.

2.27 Pain as a sensory modality: Pain, as a submodality of somatic sensation, has been defined as a "complex constellation of unpleasant sensory, emotional and cognitive experiences provoked by real or perceived tissue damage and manifested by certain autonomic, psychological, and behavioral reactions" (Terman, 2003). Normally, nociception and the perception of pain are evoked only at pressures and temperatures extreme enough to potentially injure tissues and by toxic molecules and inflammatory mediators. These high threshold physical and noxious chemical stimuli are detected by specialized peripheral sensory neurons (nociceptors). Noxious stimuli include damaging or potentially damaging stimuli including extremes of temperature, mechanical stimulation and allogens that provoke an avoidance response (Dubin, 2010). It is clear that the phenomenon of pain perception is part and parcel of the exteroceptive stimuli similar to that of other types. Thus, describing pain as a modality or sensibility merits removal and replaced by the term 'nociceptive stimuli'.

2.28 Paresis and paralysis: The term 'paresis' signifies a state of partial paralysis that is usually an upper motor neuron lesion (UMN) displaying signs such as increased tone and reflexes and is generally referred to as a state of 'spasticity'. The term 'paralysis' signifies complete loss of function and is of the lower motor neuron type (LMN) and referred to as a state of 'flaccidity'. Effects of a paresis are therefore reversible to a variable degree while effects of paralysis are generally irreversible in practice. It is therefore important to use the terms in the proper sense rather than to use the term paralysis interchangeably for both UMN and LMN lesions.

2.29 Ptosis and partial-ptosis: Partial-ptosis is the partial closure of the palpebral fissure seen following a cervical or intra-cranial lesion affecting the intracranial sympathetic pathway. Ptosis is the complete closure of the palpebral fissure due to a lesion of Cranial nerve III. The terms must not be used synonymously.

2.30 'QRS' in Electrocardiography: The 'QRS' segment or ventricular complex in common use actually begins 
normally as a ' $q$ ' wave in the left ventricular leads. The ' $q$ ' wave represents the depolarization of the interventricular septum from left-to-right and thus the electrical vector is registered as a small negative deflection named the septal 'q' wave recorded in normal conditions in aVL, Lead I, Lead II, V5 and V6 (left ventricular) leads as the wave is moving away from those leads. This is followed by a tall positive ' $R$ ' wave in these leads. Thus, the deflection wave is ' $q R$ ' in these leads (Schamroth, 1996). At the same time, the right ventricular leads (Lead III, V1 and V2) will show a 'rS' complex with no ' $q$ '. Thus, the letters Q and R reflect large deflections of the smaller and normal ' $q$ ' and ' $r$ ' waves respectively. The term ' $q$ ' indicates a normal septal depolarization sequence and ' $Q$ ' in that comparative analogy denotes a pathological wave that is used to describe the 'current of injury' or 'cavity potential' in myocardial infarction and designated as a ' $Q$ ' wave, and is not representative of a normal septal event. Thus, use of the uppercase letter ' $Q$ ' denotes a pathological activity reflecting acute myocardial injury. On a normal ECG, left ventricular leads show a 'qR' pattern while simultaneously, the right ventricular leads show a 'rS' pattern (Braunwald, 1992). This is referred to as 'reciprocal' change. There is never a deep ' $Q$ ' in any normal state of cardiovascular function, and the letter ' $Q$ ' should only be used to reflect an 'infarct' or 'current of injury'. Hence, the term "QRS" is better replaced with the term "ventricular complex" under normal conditions. The presently used form of QRS is in reality represented typically by the ' $\mathrm{rS}$ ' wave in $\mathrm{V} 1$, 'RS' in V3, 'Rs' in V4, 'RR' and 'qRs' wave in V5 and V6. 'q' waves are not seen in all leads and thus to label ventricular events with a standard 'QRS' is incorrect, and the term "ventricular complex" in context is authentic and more appropriate.

2.31 Respiratory rate: This term is universally used to determine the rate of breathing. Respiration is a general term that includes external breathing and internal (tissue) breathing, where the external denotes a purely mechanical process of diaphragmatic and chest wall mechanics, and the internal, a purely chemical process that determines energy kinetics at the cellular level. The physical test that is incorrectly termed the 'respiratory rate' is in fact a visual estimation of chest wall incursions and should be correctly referred to as the 'breathing or ventilatory rate'.

2.32 Saltatory conduction of action potential: Saltatory conduction (from the Latin 'saltare', to hop or leap) has been described as the discontinuous propagation of the action potential along myelinated axons from one node of Ranvier to the next, increasing the conduction velocity of the nerve impulse. Conduction of the impulse per-se is not intermittent, but progressive stimulation of nodal ionic entry-exit producing the action potential have been suggested. The action potential is not conducted along the myelin sheath but is a progressive phenomenon of sequential activation of membrane potential along the nodal sites of a myelinated fiber. Saltatory 'phenomenon' in peripheral myelinated axons is thus better described as 'saltatory propagation of nodal stimulation' rather than 'saltatory conduction of the action potential'. Evidence on the contrary shows that an action potential is produced by each and every one point of the internodes (Lorente, 1947), (Laporte, 1951). The conclusion, however, is in sharpest disagreement with the saltatory hypothesis (Hodgkins, 1951) and (Tasaki, 1959), according to which the action potential is produced solely by the nodes of Ranvier. During conduction, the EMF of the action potential is established successively at each and every one point of the internodes. The parametric dependence of the "Lillie transition" from continuous to saltatory conduction, up to a point, may be useful in understanding evolutionary and developmental steps in producing saltatory conduction. It shows that neither paranodal sealing nor channel clustering are absolute requirements for saltatory conduction. However, it remains unrealistic in that it has utilized a fully-formed insulating sheath that is tightened around a fully conducting axon (Young, 1993). It is in reality a progression of an intermittent nodal stimulation of the signal that drives neural transmission akin to relay stations that progressively accelerate the current flowing in electrical pylons over long distances. If the term 'saltatory conduction' must be used, then it should categorically state the phenomenon as the "saltatory propagation of nodal stimulation". Use of the term saltatory conduction to describe a generalized conduction phenomenon needs to be abandoned.

2.33 Sella turcica \& Pituitary fossa: These two terms though often used interchangeably, are not identical in reality. The sella turcica with the pituitary fossa at its center, is formed anteriorly by the tuberculum sellae including the middle clinoid processes, and posteriorly by the dorsum sellae and the posterior clinoid processes. This entire area forms the sella (Gray's Anatomy, 1995). Thus, the fossa for the pituitary gland should be referred to as the 'pituitary fossa' or 'hypophyseal fossa', and the area around the pituitary fossa as described above should be termed 'sella turcica'.

2.34 Uterine ligaments: There are two categories of commonly described ligaments in relation to the uterus that are classically described as 'true' and 'false'. The true ligaments comprise of the pubo-cervical, transverse cervical and utero-sacral components that are condensations of the endopelvic fascia. The endopelvic fascia attaches the urinary bladder, urethra, vagina and the uterus to the pelvic walls. This fascia lies immediately 
beneath the peritoneum and is one continuous unit with various thickenings or condensations in specific areas. The endopelvic fascia is continuous with the visceral fascia which provides an incomplete capsule covering the organs and allows displacements and changes in volume (Norton, 1993). Based on the origin and relationships of the true supports of the uterus, they are better described as the 'pubo-cervical fascia', 'transverse or lateral uteropelvic fascia' and 'utero-sacral fascia' respectively. Use of the term 'ligament' in context is inappropriate and incorrect. The false ligaments comprise of the round ligaments of the uterus and ovaries, and the broad ligaments which are peritoneal folds or mesentery that facilitate transmission of blood vessels, nerves and lymphatics between the pelvic wall and these organs. Based on the anatomical structure and composition, the broad ligament would be better described as the 'Utero-salpingeal mesentery'. The 'suspensory ligament' of the ovary is similarly a condensation of connective tissue connecting the lateral pelvic wall to the ovary and the salpingeal infundibulum and provides a conduit for blood vessels, nerves and lymphatics. It is therefore more appropriate to name the suspensory ligament as the 'infundibulo-pelvic' or 'ovario-pelvic fascia'.

2.35 'Wrist drop': This surprisingly innocuous term has been in use for decades and appears in both anatomical as well as clinical textbooks without any logical or scientific validity. In this related clinical scenario where temporary or permanent loss of nerve function related to the 'radial nerve' occurs with paralysis of forearm extensor muscles, the hand adopts a posture that deviates it towards the flexor compartment of the forearm and is thus in a state of apparent 'flexion'. This state is brought about by the unopposed action (tone) of the long flexors of the anterior compartment of the forearm with their preserved innervation via the median nerve to collectively bring the hand to adopt a position of flexion at the wrist. The wrist interestingly, remains in its normal position without any change. Thus, the term 'wrist drop' being incorrect as it is, needs to be replaced with the term 'hand drop' or 'acute neurogenic flexion deformity of the hand'.

\section{Conclusion}

"Science should be testable, refutable and falsifiable". Karl Popper

The importance of terminology is often underestimated. The language we use in our professional workplace underpins the way in which we communicate in the technical environment, and is not only indicative of our judgmental capabilities, but also of the organisation that we represent. Terminology is a necessity for all professionals involved in the representation, expression, communication and teaching of technical and scientific knowledge. Understanding novel words and concepts is important especially in content areas in science and medicine. Science is a discipline that relies heavily on students' ability to understand new terms and concepts (Cohen, 2012). Discussions of both initial knowledge and new knowledge which resemble the contents of the repository of medical terminology are confronted by the issue that there isn't universal agreement as to the meaning and use of terms. Since science intends to be a coherent view of the academic world, its use of terms is expected to show a logical structure with a fair degree of internal consistency. New relationship must be established between the word or term that is challengeable and devoid of logic by replacing them with new terms that are scientifically valid, semantically precise as per the intended formal scientific meaning and reference. The effort is built on ideas from use of logical semantics and the philosophy of science. The approach is supposed to provide a cogent directive towards use of appropriate and correct terminology. Science is a progressively changing domain and hence, change is an integral part of science. What was established historically, might have to change with time and this is brought to light by a memorable quote by Sir John Hunter who is known to have said, "Never ask me what I have said or what I have written: but if you will ask me what my present opinions are, I will tell you" (Causey, 1955).

\section{References}

Allan DW, Greer JJ, 1997. Embryogenesis of the phrenic nerve and diaphragm in the fetal rat. J. Comp. Neurol. 382, 459468

Babiuk RP, Zhang W, Clugston R, Allan DW, Greer JJ, 2003. Embryological origins and development of the rat diaphragm. $J$. Comp. Neurol. 455, 477-487

Braunwald E. 1992. Heart Disease: Textbook of Cardiovascular Medicine. Vol 1. W. B. Saunders. Ch.5: 122

Brooks EG, Reed KD. 2015. Principles and Pitfalls: a Guide to Death Certification. Clin Med Res. 2015 Jun; 13(2): 74-82. doi: $10.3121 / \mathrm{cmr} .2015 .1276$

Causey, G. 1955. The Functional Importance of the Blood Supply of Peripheral Nerve. Ann R Coll Surg Engl. 1955 Jun; 
16(6): 367-383.

CDC. 2003. Physicians' Handbook on Medical Certification of Death. Department of Health and Human Services. National Center for Health Statistics. DHHS Publication No. (PHS) 2003-1108. https://www.cdc.gov/nchs/data/misc/hb_cod.pdf

Chakravarty M, Shome DK, Sarwani NAL, James H, Al Tabban MY. 2018. Acquired Immune Deficiency Syndrome Renaming a Scientific impropriety. Journal of Education and Practice Vol.9, No. 26, pp74-79.

Cohen Marisa T. 2012. Kappa Delta Pi record. The Importance of Vocabulary for Science Learning. v48 n2 p 72-77. https://eric.ed.gov/?id=EJ993005

Conze J, Klinge Uwe, Schumpelick V. 2001. Surgical Treatment: Evidence-Based and Problem-Oriented Hernias. University Hospital, Department of Surgery, RWTH, Aachen, Germany

de Astudillo LR, Niaz M. 1996. Reasoning strategies used by students to solve stoichiometric problems and its relationship to alternative conceptions, prior knowledge and cognitive variables. J Sci Educ Technol, 5: 131-140. https://doi.org/10.1007/BF01575153.

Dubin AE, Patapoutian A. 2010. Nociceptors: the sensors of pain pathway. J Clin Invest. 120(11): 3760-3772. doi: $10.1172 / \mathrm{JC} 142843$.

Fardon DF, Williams AL, Dohring EJ, Murtagh FR, Gabriel Rothman SL, Sze GK. 2014 Lumbar disc nomenclature: version 2.0: Recommendations of the combined task forces of the North American Spine Society, the American Society of Spine Radiology and the American Society of Neuroradiology. Spine J. 14 (11): p. 2525-45. doi: 10.1016/j.spinee.2014.04.022

FEDERATIVE INTERNATIONAL PROGRAM ON ANATOMICAL TERMINOLOGIES. 2013. Footnote TA98. http://www.unifr.ch/ifaa/Public/EntryPage/TA98\%20Tree/Entity\%20TA98\%20EN/04.0.00.003\%20Entity\%20TA98\%2 0EN.htm

Fitzgibbons RJ, Jr, Forse RA. 2015. Clinical practice. Groin hernias in adults. N Engl J Med. 372 (8): pp 756-63. doi:10.1056/NEJMcp1404068. PMID 25693015

Goyal RK, Bauer JL, Spiro HM. 1971. The nature and location of lower esophageal ring. N Engl J Med; 284(21):1175-1180

Gray's Anatomy, 1995, 38th Edition, ISBN 0-443-04560-7, Churchill Livingstone, p 585-6

Gray's Anatomy, 1995, 38th Edition, ISBN 0-443-04560-7, Churchill Livingstone. p 499

Haldeman S. 1990. American Spine Society: failure of pathology model to predict back pain. Spine. 15(7): 718-24.

Hicks J. 1953. The mechanics of the foot. The joints. J Anat, 87, pp. 345-357

Hohe J, Gerard Ateshian, Maximilian Reiser, Karl-Hans Englmeier, and Felix Eckstein, 2002. Surface Size, Curvature Analysis, and Assessment of Knee Joint Incongruity with MRI In Vivo. Magnetic Resonance in Medicine 47:554-561. DOI 10.1002/mrm.10097

Hodgkin, AL, 1951. THE IONIC BASIS OF ELECTRICAL ACTIVITY IN NERVE AND MUSCLE. Biol. Rev. $26,339$. https://doi.org/10.1111/j.1469-185X.1951.tb01204.x

Hughes IA, Acerini CL. 2008. Factors controlling testis descent. Eur J Endocrinol; 159 (Suppl.1): 75-82

John Wiley \& Sons. Inc. 1997. J Res Sci Teach 34: 655-660, 1997.

Johnstone AH. 2000. Teaching of Chemistry - Logical or Psychological? A.H. Johnstone, Chem. Edu. Res. Pract. Eur. 1, 915

Kitab SA, Miele VJ, Lavelle WF, Benzel EC. 2009. Pathoanatomic basis for stretch-induced lumbar nerve root injury with a review of the literature. Neurosurgery. 65(1):161-7; discussion 167-8. doi: 10.1227/01.NEU.0000347002.67982.8F.

Kwan MK, Wall EJ, Massi J, Garfin SR. Published 2009. Stress and stretch of peripheral nerve Rabbit experiments in vitro and in vivo. Acta Orthopedica Scandinavica. 1992 Vol 63 (3) https://doi.org/10.3109/17453679209154780.

Laporte YJ. 1951. Role of Potential Wave Spreading Along Myelinated Nerve Fiber in Excitation and Conduction. Gen. Physiol., 35, 323

Logan SR. 1998. Physical Chemistry for Biomedical Sciences. Taylor \& Francis, 1998, p-11

Lorente De Nó and V. Honrubia. 1964. Continuous conduction of action potentials by peripheral myelinated fibers. Proc Natl Acad Sci U S A. 52(2): 305-312) PMCID: PMC300276, PMID: 14206595

Merrell, A.J., Ellis, B.J., Fox, Z.D., Lawson, J.A., Weiss, J.A., Kardon, G., 2015. Muscle connective tissue controls development of the diaphragm and is a source of congenital diaphragmatic hernias. Nat. Genet. 47, 496-504. 
Merriam-Webster. Aponeurosis. https:/www.merriam-webster.com/dictionary/aponeurosis

Merriam Webster dictionary

https://www.google.com.bh/search?q=ganglia+definition\&oq=ganglia + definition\&aqs $=$ chrome..69i57j015.7083j0j1\&s ourceid=chrome $\&$ ie $=\mathrm{UTF}-8$

Muscolino, J. 2010. The Muscular System Manual. The Skeletal Muscles of the Human Body. 3rd Edition. eBook ISBN: 9780323071352. Mosby's Essential Sciences for Therapeutic Massage: Anatomy, Physiology. By Sandy Fritz.

NCI Dictionary of Cancer Terms. https://www.cancer.gov/publications/dictionaries/cancer-terms/def/malignancy.

Rubin R, Strayer DS. 2012. Rubin's Pathology. Clinicopathologic Foundations of Medicine. $6^{\text {th }}$ Ed. Lippincott Williams \& Wilkins, Ch 5, p 161-162

Sampaio FJB, Favorito LA. 1998. Analysis of testicular migration during the fetal period in humans. J Urol; 159: 540-542

Santen RJ, Allred DC, Ardoin SP, Archer DF, Boyd N, Braunstein GD, Burger HG, Colditz GA, Davis SR, Gambacciani M, Gower BA, Henderson VW, Jarjour WN, Karas RH, Kleerekoper M, Lobo RA, Manson JE, Marsden J, Martin KA, Martin L, Pinkerton JV, Rubinow DR, Teede H, Thiboutot DM, Utian WH. 2010. Postmenopausal hormone therapy: An Endocrine Society scientific statement. J. Clin. Endocrinol. Metab. 95 (7 Suppl 1): s1-s66. doi:10.1210/jc. pp 2009-2509.

Sarrafian SK. 1993. Biomechanics of the subtalar joint complex. Clin Orthop Relat Res; 290: pp 17-26.

Schamroth, L. 1996. An Introduction to Electrocardiography. Blackwell Science, 7th Ed. pp18-19

Schwab M. 2011. Hepatic Acinus. (eds) Encyclopedia of Cancer. Springer, Berlin, Heidelberg. https://doi.org/10.1007/978-3$642-16483-5$

Scott-Conner CEH, Dawson DL. 1993. Operative Anatomy. J.B. Lippincott Co. Philadelphia. ISBN 0-397-51007-1

Sefton EM, Gallardo M, Kardon G. 2018. Developmental origin and morphogenesis of the diaphragm, an essential mammalian muscle. 2018. Developmental Biology 440 (2018) 64-73. https://doi.org/10.1016/j.ydbio.2018.04.010).

Seringe R, Wicart P. 2013. Orthopaedics \& Traumatology: Surgery \& Research. Volume 99, Issue 6, Supplement, pp 345-355. https://doi.org/10.1016/j.otsr.2013.07.003

Shwartz. Principles of Surgery. 1999. 7th Ed, McGraw-Hill, Vol 2. Ch 34, p 1535.

https://www.laserspineinstitute.com/herniated_disc/differences-between-herniated-ruptured-slipped-disc-protrusion/

Slisco J. Dewey I, Dykstra Jr. 1998. The role of scientific terminology in research and teaching: Is something important missing? J Res Sci Teach 34: 655-660 https://doi.org/10.1002/(SICI)1098-2736(199708)34:6<655::AIDTEA7>3.0.CO;2-M

Stuenkel CA, Davis SR, Gompel A, Lumsden MA, Murad MH, Pinkerton JV, Santen RJ. 2015. "Treatment of Symptoms of the Menopause: An Endocrine Society Clinical Practice Guideline". J. Clin. Endocrinol. Metab. 100 (11): 3975-4011. doi:10.1210/jc.2015-2236

Tasaki I. 1959. Handbook of Physiology, 1, 75. Washington, D. C.: American Physiological Society. (Huxley, A. F., and R. StAmpffi, J. Physiol., 108, 315

Terman GW, Bonica JJ. 2003. Spinal mechanisms and their modulation. In: Loeser JD, Butler SH, Chapman CR, Turk DC, eds. Bonica's Management of Pain. 3rd ed. Philadelphia, Pennsylvania, USA: Lippincott Williams and Wilkins; 2003:73

The Free Dictionary. https://medical-dictionary.thefreedictionary.com/aponeurosis

Tobin RW. Esophageal rings, webs, and diverticula. J Clin Gastroenterol 1998; 27(4):285-295

Varadarajulu S, Noone T. 2003. Symptomatic lower esophageal muscular ring: response to Botox. Dig Dis Sci; 48(11):21322134

Young, RG, Castel-franco AM, Hartline, DK. 1993. The "Lillie Transition": Models of the Onset of Saltatory Conduction in Myelinating Axons. Békésy Laboratory of Neurobiology. Pacific Biosciences Research Center. University of Hawaì 1 at Manoa, Honolulu, HI 96822 https://core.ac.uk/download/pdf/10599258.pdf 